\title{
ER membrane protein complex subunit 6 (EMC6) is a novel tumor suppressor in gastric cancer
}

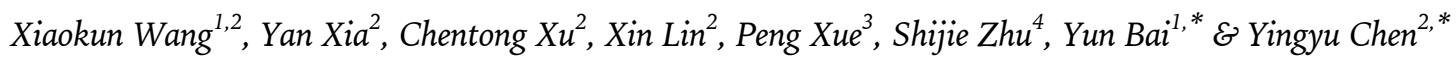 \\ Departments of ${ }^{1}$ Cell Biology and ${ }^{2}$ Immunology, School of Basic Medical Science, Peking University, Beijing 100191 , ${ }^{3}$ College of \\ China-Japan Friendship Hospital, Beijing University of Chinese Medicine, Beijing 100029, ${ }^{4}$ Department of Oncology, Wangjing Hospital, \\ China Academy of Chinese Medical Sciences, Beijing 100102, China
}

\begin{abstract}
The endoplasmic reticulum (ER) membrane protein complex subunit 6 (EMC6) is a novel human autophagy-related molecule. Here, using tissue microarray and immunohistochemistry, we report that EMC6 protein is lost or reduced in glandular cells of patients with gastric adenocarcinoma, compared to normal stomach mucosa. Overexpression of EMC6 in gastric cancer cells inhibited cell growth, migration, invasion, and induced apoptosis and cell cycle arrest at S-phase. Further investigation suggested that EMC6 overexpression in BGC823 human adenocarcinoma gastric cancer cells reduced tumorigenicity in a xenograft model, demonstrating that EMC6 has the characteristics of a tumor suppressor. This is the first study to show that EMC6 induces cell death in gastric cancer cells. The molecular mechanism of how EMC6 functions as a tumor suppressor needs to be further explored. [BMB Reports 2017; 50(8): 411-416]
\end{abstract}

\section{INTRODUCTION}

The endoplasmic reticulum (ER) protein membrane complex subunit 6 (EMC6), also known as transmembrane protein 93 (TMEM93), is a human autophagy-related protein that we identified in our laboratory (1). The EMC6 gene is located on human chromosome 17p13.2, and includes 2 exons and 1 intron. The EMC6 ORF (open reading frame) encodes a predicted $12.2 \mathrm{kDa}$ protein of 110 amino acids with an isoelectric point of 10.08. EMC6 is a transmembrane protein, and has two transmembrane domains in the amino acid

*Corresponding authors. Yun Bai, Tel: +86-82801495; Fax: +8610-82801149; E-mail: baiyun@bjmu.edu.cn, Yingyu Chen, Tel: +86-10-82802846-420; Fax: +86-10-82801149; E-mail: yingyu_ chen@bjmu.edu.cn

https://doi.org/10.5483/BMBRep.2017.50.8.065

Received 19 April 2017, Revised 11 May 2017, Accepted 23 June 2017

Keywords: Anti-tumor activity, Apoptosis, Cell cycle, EMC6, Gastric cancer regions 48-68 and 87-107. EMC6 is conserved and widely expressed in a variety of human tissues and organs, and some cancer cell lines. EMC6 protein is located in the ER $(1,2)$. Overexpression of EMC6 in U2OS osteosarcoma and HCT116 colon carcinoma cells induces autophagosome formation and promotes the degradation of autophagic substrates in the lysosome (1). EMC6 interacts with RAB5A and Beclin-1 and recruits $R A B 5 A$ to the $E R$, thereby increasing the binding and activity of RAB5/Phosphatidylinositol 3-kinase catalytic subunit type 3 (PIK3C3) complexes, and promotes autophagosome formation (1). Shen et al. (3) reported that overexpression of EMC6 increases autophagy in glioma cell lines, inhibits the proliferation of glioma cells, suppresses tumorigenicity of glioma cells in brain, and prolongs the survival time of nude mice challenged with glioma cells. Evidence from C. elegans studies shows that EMC6 activity is involved in the maturation, expression, and stability of levamisole-sensitive acetylcholine receptors (L-AChRs), which play an important role in maintaining homeostasis of the ER (4). Knockout of emc6 gene leads to the death of nematode embryos, suggesting that EMC6 is critical during development. Up to now, there have been no studies detailing the involvement of EMC6 in human disease.

In this study, we used tissue microarray and immunohistochemistry to demonstrate that EMC6 protein expression is either reduced or lacking in gastric cancer. Restoration of EMC6 expression inhibits gastric cancer cell growth, induces apoptosis, and causes cell cycle arrest at $\mathrm{S}$ phase, suggesting that EMC6 has significant anti-tumor activity. This is the first study to clarify the biological activities of EMC6, and provides the basis to explore future applications of EMC6 in cancer biology.

\section{RESULTS}

EMC6 protein is under-expressed in gastric adenocarcinoma cells and EMC6 overexpression inhibits growth of gastric cancer cells

Through searching the EMC6 information database in The Human Protein Atlas (THPA) website (http://www.proteinatlas. org/ENSG00000127774-EMC6/tissue/stomach), we observed that EMC6 mRNA and EMC6 protein are moderately expressed

ISSN: 1976-670X (electronic edition)

Copyright (C) 2017 by the The Korean Society for Biochemistry and Molecular Biology

(c) This is an open-access article distributed under the terms of the Creative Commons Attribution Non-Commercial License (http://creativecommons.org/licenses/by-nc/4.0) which permits unrestricted non-commercial use, distribution, and reproduction in any medium, provided the original work is properly cited. 
in normal gastric tissue, but display loss or low expression in gastric cancer tissue. Using tissue microarray and immunohistochemistry, the expression of EMC6 protein in non-tumor tissue adjacent to gastric cancer and gastric adenocarcinoma tissue was analyzed. EMC6 protein showed moderate or high expression levels in most non-tumor tissues adjacent to cancer (Fig. S1A). It was mainly located in the cell cytoplasm of the mucosa gland, and had a diffuse expression pattern. However, EMC6 protein expression was reduced or undetected in gastric adenocarcinoma cells (Fig. S1A). These results are consistent with the THPA analysis of EMC6, and suggest that EMC6 may have an inhibitory effect in the development of gastric cancer.

We used the Kaplan-Meier Plotter online database (http:// kmplot.com/analysis/index.php? $\mathrm{p}=$ service\&cancer $=$ gastric) (5) to determine the correlation between EMC6 mRNA levels and survival time in 876 patients with gastric cancer. High levels of EMC6 mRNA correlated with better overall survival in gastric cancer patients (Fig. S1B), indicating that elevated expression of EMC6 may be a favorable prognostic indicator in patients with gastric cancer.

Next, the biological consequences of ectopic expression of EMC6 on the growth and viability of gastric cancer cell lines were evaluated. EMC6 protein expression was significantly increased in a dose-dependent manner in BGC823 cells infected with Ad5-EMC6 (Fig. 1A). The MTS cell proliferation assay indicated that Ad5-EMC6 infection of BGC823 cells resulted in significant growth inhibition, compared to Ad5-Null infection (Fig. 1B, C). This growth inhibition was time- and dose-dependent. This anti-proliferative effect was further demonstrated in a colony formation assay, as EMC6 overexpression significantly suppressed the colony-forming ability of BGC823 cells (Fig. 1D, E). Similar results were observed in SGC7901 human gastric cancer cells (Fig. S2).
To investigate the influence of overexpression of EMC6 on gastric cancer proliferation in vivo, BGC-823 cells stably overexpressing EMC6 were established, These cells exhibited significantly increased EMC6 expression compared to cells transfected with an empty control vector (Fig. 1F). BGC823 cells transfected with an empty vector or BGC823 cells stably overexpressing EMC6 were injected into the right axilla of BALB/C nude mice. Mice were sacrificed at 15-20 days after inoculation; the tumors were excised and photographed. Mice injected with stable EMC6-overexpressing BGC823 cells had smaller tumors than those injected with BGC823 cells containing an empty vector (Fig. 1G). About one quarter to one third of the mice specifically injected with stable EMC6-overexpressing BGC823 cells had no tumor growth, indicating that EMC6 reduced the tumorigenicity of BGC823 cells.

\section{EMC6 overexpression induces apoptosis involving activation of the caspase cascade}

It has previously been demonstrated that EMC6 overexpression in human U2OS osteosarcoma and HCT116 colon carcinoma cells induces autophagic features (1). We investigated whether EMC6-mediated autophagy occurred in BGC823 cells or SGC7901 cells. As shown in Fig. 2A, Fig. S3A and B), overexpression of EMC6 failed to upregulate LC3B-II accumulation under normal nutrient conditions, when compared with the vector control group. Chloroquine (CQ) is a known inhibitor of the late phase of autophagy (6). CQ inhibits autophagy as it raises the lysosomal $\mathrm{pH}$, which leads to inhibition of both fusion of autophagosome with lysosome and lysosomal protein degradation. After treatment with $C Q$, the accumulation of the LC3B-II protein was increased in both EMC6- and empty vector-transfected cells (Fig. 2A, Fig. S3A
A

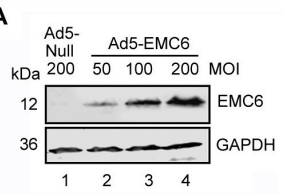

D

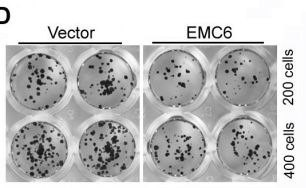

B

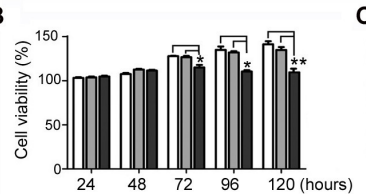

E

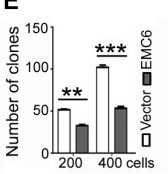

$\mathbf{F}$

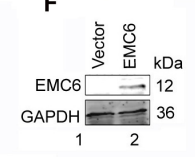

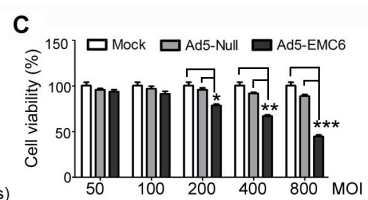

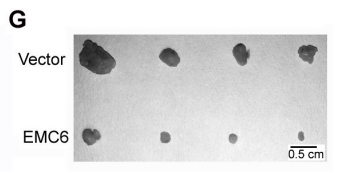

Fig. 1. EMC6 induces growth arrest of BGC823 cells. (A) BGC823 cells were infected with either Ad5-EMC6 or Ad5-Null at the indicated $\mathrm{MOI}$ for $24 \mathrm{~h}$. The dose-dependent expression of EMC6 was analyzed by western blotting. (B) BGC823 cells were infected with either Ad5-EMC6 or Ad5-Null at $200 \mathrm{MOI}$ for the indicated time. Cell viability was detected by MTS assay. (C) BGC823 cells were infected with either Ad5-EMC6 or Ad5-Null at different MOI for $72 \mathrm{~h}$. Cell viability was detected by MTS assay. (D) Representative images of the colony formation in BGC823 cells transfected with pCDB-EMC6 or pCDB-Vector were shown. (E) Cells were treated as (D), the number of clones was counted and the data were expressed as mean \pm SEM from 3 experiments. (F) Western blotting analysis of the expression of EMC6 in BGC823 cells stable transfected with pCDB-Vector or pCDB-EMC6. (G) pCDB-Vector or pCDB-EMC6 stable transfected BGC823 cells were injected subcutaneously in BALB/C nude mice. Excised xenograft tumors were imaged on day 15 . $* P<<0.05, * * P<0.01$, $* * * \mathrm{P}<0.001$ 


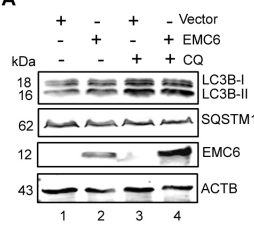

B

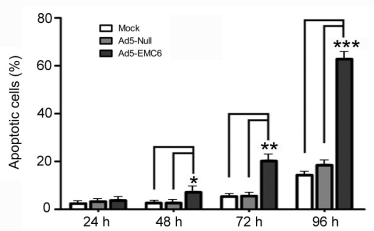

C

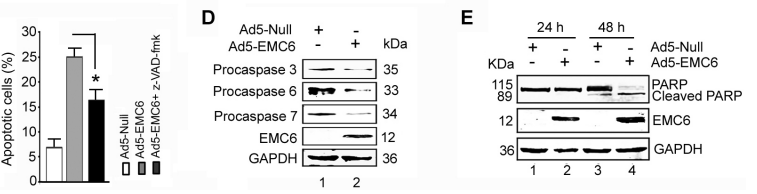

Fig. 2. EMC6 induces cell apoptosis, not autophagy. (A) BGC823 cells were transfected with either pCDB-Vector or pCDB-EMC6 for $24 \mathrm{~h}$, and then treated with CQ $(25 \mu \mathrm{M})$ at the last $4 \mathrm{~h}$. The levels of endogenous LC3B-II and SQSTM1 protein were analyzed by Western blotting. (B) BGC823 cells were infected with either Ad5-Null or Ad5-EMC6 at $200 \mathrm{MOI}$ for the indicated time, the cell apoptosis was measured by FITC-Annexin V/PI staining and flow cytometry. The number of apoptotic cells included Annexin $\mathrm{V}^{+} \mathrm{PI}^{+}$double and Annexin $\mathrm{V}^{+}$single positive cells. Data were expressed as the mean \pm SEM of the results from 3 experiments. (C) BGC823 cells were pretreated with $40 \mu \mathrm{M}$ of $\mathrm{z}$-VAD-fmk for $2 \mathrm{~h}$, and then infected with Ad5-EMC6 for another $72 \mathrm{~h}$. Apoptotic cells were analyzed by flow cytometry. (D) BGC823 cells were infected with either Ad5-Null or Ad5-EMC6 for $24 \mathrm{~h}$, the levels of endogenous procaspase- $3,-6$, and -7 were analyzed by western blotting. (E) BGC823 cells were infected with either Ad5-Null or Ad5-EMC6 for $24 \mathrm{~h}$ or $48 \mathrm{~h}$, the levels of endogenous PARP protein were analyzed by western blotting. ${ }^{*} \mathrm{P}<$ $0.05, * * P<0.01, * * * P<0.001$

and B, lane 4 vs. lane 3), but there is no obvious difference between these two groups. Consistent with the results of western blotting, there was no change in the distribution of GFP-LC3B dots between EMC6 and empty vector-transfected cells in the presence or absence of CQ (Fig. S3C). Simultaneously, the endogenous autophagic substrate P62/SQSTM1 (Sequestosome-1) did not change in either EMC6- or control vector-transfected cells (Fig. 2A, Fig. S3A and $\mathrm{B})$. These data suggest that EMC6 failed to induce autophagy in BGC823 and SGC7901 cells, indicating that EMC6-mediated autophagy may be cell type specific.

Apoptosis is just one of many factors that can affect cell viability. Therefore, we evaluated the exposure of phosphatidylserine (PS) asymmetry, a key biochemical hallmark of apoptosis, in cell lines infected with either Ad5-Null or Ad5-EMC6. Propidium iodide (PI) staining was employed to assess plasma membrane integrity. Data from experiments indicated that Ad5-EMC6 infection resulted in a significant time-dependent increase in the number of apoptotic cells compared to mock infection with Ad5-Null (Fig. 2B and Fig. S4).

To determine whether EMC6 overexpression enhanced apoptosis via the caspase-dependent pathway, BGC823 cells were pretreated with the pan-caspase inhibitor, z-VAD-fmk, for
A
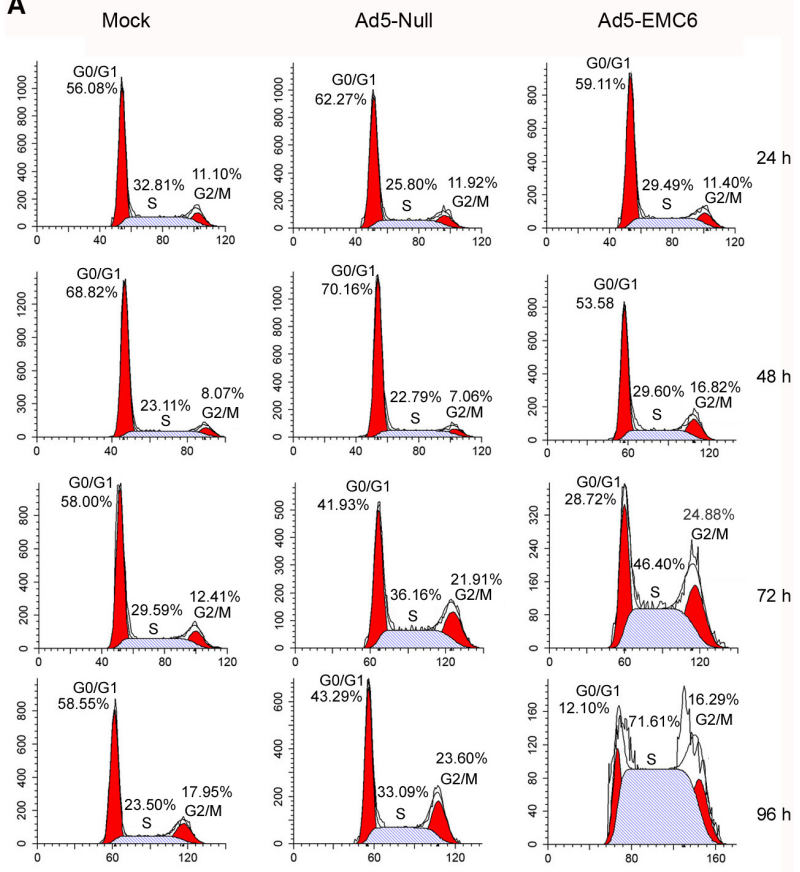

B

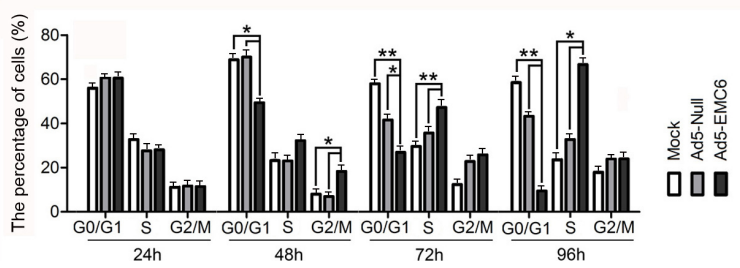

Fig. 3. EMC6 induces cell cycle arrest at S-phase. (A) BGC823 cells were infected with either Ad5-Null or Ad5-EMC6 at 200 $\mathrm{MOI}$ for different time; the percentage of cells in each phase of the cell cycle was measured by PI staining and flow cytometry. (B) Data are expressed as the mean \pm SEM of the results from 3 experiments. $* P<0.05, * * P<0.01, * * * P<0.001$.

$2 \mathrm{~h}$ and were then infected with Ad5-EMC6 for $72 \mathrm{~h}$. Flow cytometric analysis revealed that pre-treatment with z-VADfmk partly reduced the percentage of apoptotic cells (Fig. 2C). Western blotting analysis confirmed that the levels of procaspase-3, procaspase- 6 , and procaspase- 7 were significantly decreased in Ad5-EMC6 infected cells at $24 \mathrm{~h}$, compared with that of Ad5-Null-infected cells (Fig. 2D). We next detected the cleavage of Poly (ADP-ribose) polymerase (PARP), a substrate of caspase-3. There was no difference in cleaved PARP between Ad5-Null and Ad5-EMC6-infected cells at $24 \mathrm{~h}$ (Fig. 2E). However, the levels of cleaved PARP were significantly increased in Ad5-EMC6 infected cells at $48 \mathrm{~h}$, compared with the Ad5-Null group (Fig. 2E). These data demonstrate the activation of the caspase-dependent apoptosis pathway, which partially mediated EMC6-induced cell apoptosis in EMC6overexpressing BGC823 cells. 
EMC6 overexpression induces S-phase cell cycle arrest Based on the preliminary assays in which we determined the effects of EMC6 on cell viability and apoptosis, the effect of EMC6 on cell cycle progression was also explored. After removing data artifacts indicating dead cells and cellular debris, we determined the percentage of cells in each group in a particular stage of the cell cycle (G0/G1, S, and G2/M). A much higher percentage of cells in S-phase was observed in Ad5-EMC6 infected BGC823 cells than in the Ad5-null and mock groups. This increase in the percentage of cells in S-phase occurred in a time-dependent manner (Fig. 3A and B). Compared with the control group, the percentages of cells in S-phase among Ad5-EMC6-infected BGC823 cells at $24 \mathrm{~h}$ to $96 \mathrm{~h}$ increased from $29.49 \%$ to $29.60 \%$, then to $46.40 \%$, and finally to $71.61 \%$. In each case, there was a concomitant reduction in the proportion of cells in the G0/G1 phases. This data suggest that inhibition of cell growth and induction of cell death in EMC6-overexpressing cancer cells may be associated with the induction of S-phase cell cycle arrest.

EMC6 overexpression decreases the migration and invasion We investigated whether EMC6 overexpression affected migration and invasion of gastric cancer cell lines. Ectopic overexpression of EMC6 in BGC823 cells dramatically inhibited cell migration (Fig. $4 \mathrm{~A}$ and B). Similar results were observed in SGC7901 cells (Fig. S5). Moreover, ectopic overexpression of EMC6 in BGC823 cells also inhibited invasion in transwell Matrigel invasion assays (Fig. 4 C and D). These findings demonstrated that overexpression of EMC6 reduced the motility of gastric cancer cells, and suggest that EMC6 may play a crucial role in metastasis of human gastric cancer cells.

\section{DISCUSSION}

In the present study, it was found that expression of EMC6 is

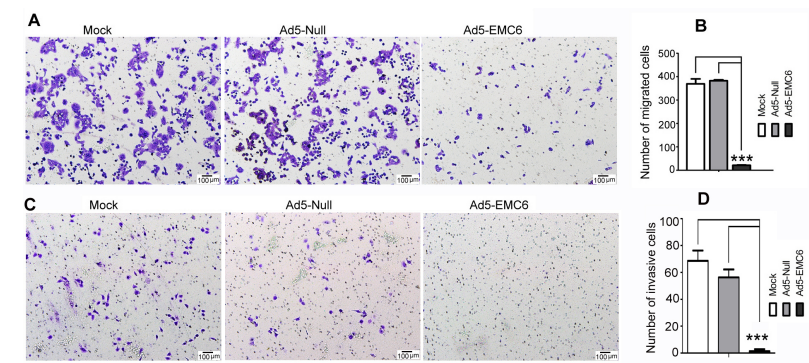

Fig. 4. Ad5-EMC6 decreases migration and invasion. (A and C) BGC823 cells $\left(1 \times 10^{5}\right.$ cells) were infected with either Ad5-Null or Ad5-EMC6 $(\mathrm{MOI}=200)$ for $24 \mathrm{~h}$, then performed for transwell assay with or without Matrigel pretreatment. Representative images of migrated and invasive cells are shown. ( $B$ and $D$ ) The number of cells migrated or invaded per field. Data are presented as the mean \pm SEM of the results from 3 experiments. $* * * P<0.001$. significantly decreased in gastric adenocarcinoma samples compared to normal gastric tissue. Moreover, high levels of EMC6 mRNA in gastric tissues correlated to better prognosis of patients with gastric cancer, indicating that the expression of EMC6 may be a potential independent prognostic factor for patients with gastric cancer. Further investigation indicated that EMC6 overexpression in the gastric cancer cells can significantly alter the biological behaviors, resulting in decreased cell viability, growth inhibition, cell cycle arrest, and induction of apoptosis. These phenotypes may occur independently in parallel pathways, or cooperatively, to lead to cell death. In addition, overexpression of EMC6 suppressed the migration and invasion of gastric cancer cells. These data for the first time suggest that overexpression of EMC6 exerted strong antitumor activity in gastric cancer cells.

The ER membrane protein complex (EMC) family contains six subunits in yeast, (7), and possibly functions to eliminate misfolded ER proteins. In humans, the EMC complex comprises ten subunits, EMC1-10 (2). This complex is thought to be involved in an ER quality control process called ERassociated degradation (ERAD) (8). Using homology-searching algorithms (9), it has been shown that the EMC is conservative in the process of evolution and has significant homology to the corresponding proteins of species ranging from yeast to mice. Recent studies suggest that the EMC has been involved in a variety of biological activities, but the molecular mechanism remains unclear. Lahiri et al. (10) suggested that the EMC may facilitate contact between ER and mitochondria by regulating protein folding or processing. The EMC is required for the stable expression of transmembrane proteins such as rhodopsin, and its loss was associated with retinal degeneration in Drosophila (11). EMC1 as a molecular chaperone, can promote the transport of SV4O virus from ER to plasma membrane, and can support viral infection (12). Using wholeexome sequencing, it was found that EMC1 gene variants, either biallelic or monoallelic, might lead to a syndrome characterized by intellectual disability and degeneration of the cerebellum (13). There is no report about the relationship between EMC family and human tumors.

Previous studies demonstrated that EMC6 is a positive regulator of autophagy. It interacts with both RAB5A and BECN1/Beclin 1, facilitates RAB5A localization of ER, and enhances autophagosome formation (1). In HCT116 colon cancer cells and U2OS osteosarcoma cells, EMC6 overexpression induced autophagy, evidenced by increased LC3B-II protein accumulation, and accelerated the degradation of autophagic substrates (1). In the glioma cell lines SHG44, U87, and U251, EMC6 suppresses cell growth via induction of autophagy (3). The xenograft model indicated that EMC6 inhibited the intracranial tumorigenicity of the U87 glioma cell line (3). In the present study, we did not observe obvious autophagic phenotypes in BGC823 and SGC7901 cells overexpressing EMC6. This finding implied that EMC6 may induce cell autophagy in a cell-type specific manner. 
Immediate studies to follow include expansion of the present study with additional cell lines to explore the molecular mechanisms that EMC6 is involved in. In addition, whether other EMC subunits can also act in autophagy or execute completely different functions remain to be clarified. The association between EMC family expression and human cancer also needs to be evaluated. In future studies we will engage in clinical investigations regarding the expression of EMC6 in gastric cancer patients, and we will analyze its correlation with clinical pathological parameters.

\section{MATERIALS AND METHODS}

\section{Cell culture and antibodies}

BGC823 and SGC7901 cells were obtained from American Type Culture Collection (ATCC) (Manassas, VA, USA) and cultured in Dulbecco's Modified Eagle Medium (DMEM) (Invitrogen, California, CA, USA) supplemented with 10\% Ausbian fetal bovine serum (Shanghai VIAN-SAGA biotech. Ltd, Shanghai, China). Plasmid transfection was performed using MegaTran 1.0 Transfection Reagent (ORIGEN, Rockville, MD, USA) according to the manufacturer's instruction. G418 was used to select cells with stably transfected with pCDBVector or pCDB-EMC6. Inhibition of apoptosis was achieved by treating cells with $40 \mu \mathrm{M}$ of z-VAD-fmk.

The following antibodies were used: Rabbit anti-human EMC6 antibody was produced from our laboratory (1); Rabbit anti-caspase 3 (Cell Signaling Technology, Danvers, MA, USA), caspase 6 and caspase 7 (Abcam, Cambridge, UK), Rabbit anti-p62/SQSTM1 antibody (MBL International, Woburn, MA, USA), anti-LC3B antibody (Sigma-Aldrich, St. Louis, MO, USA), anti-PARP antibody (Cell Signaling Technology, Danvers, MA, USA). Secondary antibodies included DyLight 800/DyLight 680-conjugated IgG against mouse or rabbit from Rockland (Limerick, PA, USA). All reagents were purchased from SigmaAldrich (St. Louis, MO, USA), unless stated otherwise.

\begin{abstract}
Adenoviral vectors
All of the recombinant adenovirus was based on type 5 (E1/E3-deficient) adenovirus. The complete coding sequence of EMC6 was subcloned into the BamHI and EcoRI sites of the pShuttle-CMV vector. The expression cassette of EMC6 was then transferred into the adenoviral backbone vector pAdxsi, and the recombinant clones of the shuttle plasmid and viral backbone plasmid of EMC6 were confirmed by DNA sequencing. The recombinant viral plasmid of EMC6 (Ad5EMC6) was then linearized by Pacl digestion and packaged in HEK293 cells. All of the viral particles were purified by cesium chloride density gradient centrifugation and titrated by the TCID50 method.
\end{abstract}

\section{Cell viability assay}

Cell viability assays were performed using the CellTiter 96 AQueous One Solution Cell Proliferation Assay (Promega,
Madison, WI, USA) according to the manufacturer's instructions. Briefly, BGC823 cells (3000 cells/well) were plated in 96-well culture plates and infected by Ad5-Null or Ad5-EMC6 at the indicated multiplicity of infection (MOI) for different durations. CellTiter 96 AQueous One Solution was added to a concentration of $10 \mu \mathrm{l} /$ well, and cells were incubated for $4 \mathrm{~h}$ and subsequently assayed on an EL-311SX ELISA Reader (Bio-Tec Instruments, Winooski, VT, USA) at $490 \mathrm{~nm}$. Cell viability was calculated as follows: cell viability $=$ absorbance of test group/absorbance of control cell group $\times 100 \%$. Each experiment was performed in biological triplicate and independently repeated four times.

\section{Colony formation assay}

Cells transfected with pCDB-Vector or pCDB-EMC6 were plated in triplicate at 200 or 400 cells/well in 24-well plates and cultured for 10-14 days. Cells were then fixed with $4 \%$ paraformaldehyde for $30 \mathrm{~min}$ and stained with crystal violet for $30 \mathrm{~min}$. Colonies containing more than 50 cells were counted and photographed. All of the experiments were repeated three times, and the average values were reported.

\section{Cell apoptosis assay}

BGC823 cells were infected with either Ad5-Null or Ad5-EMC6 for 24 to 96 h. Cell apoptosis was detected using an FITC-Annexin V/PI staining detection kit (Beijing Biosea Biotechnology Co., Ltd., China) according to the manufacturer's instruction. Fluorescence signals were detected through a FACSCalibur flow cytometer (BD Biosciences, Franklinlake, $\mathrm{NJ}$, USA) to determine the percentage of apoptotic cells, which included Annexin $\mathrm{V}^{+} \mathrm{PI}^{+}$double and Annexin $\mathrm{V}^{+}$single positive cells.

\section{Cell cycle analysis}

BGC823 cells were infected with either Ad5-Null or Ad5EMC6 for 24 to $96 \mathrm{~h}$. The cells were fixed with $70 \%$ ethanol at $-12^{\circ} \mathrm{C}$ overnight, then treated with $100 \mu \mathrm{g} / \mathrm{ml}$ RNase A for 30 min at $37^{\circ} \mathrm{C}$, and stained using $\mathrm{PI}$ in $0.2 \%$ Triton $\mathrm{X}-100$. Subsequently, the cells were collected on a FACSCalibur flow cytometer and the percentages of cells in the different phases of the cell cycle (G0/G1, S and G2/M) were analyzed using ModFit software (BD Biosciences, Franklinlake, NJ, USA). In the analysis of cell cycle, we set up the "gate", and the death cells, cell debris and aggregates were excluded.

\section{Migration and invasion assay}

In vitro migration and invasion assays were carried using 8- $\mu \mathrm{m}$ transwell inserts, either with or without BD Falcon ${ }^{\mathrm{TM}}$ Matrigel (Corning incorporated, New York, USA), respectively. Cells $\left(1 \times 10^{5}\right)$ infected with either Ad5-Null or Ad5-EMC6 $(\mathrm{MOI}=$ 200) for $24 \mathrm{~h}$ were incubated in serum-free media at the top chamber of each transwell insert, and serum-containing media was added to the lower chamber. After $24 \mathrm{~h}$, cells that migrated/invaded were fixed in $10 \%$ formalin, stained with 
$1 \%$ crystal violet, and counted under a light microscope.

\section{Western blot}

Cells were collected and extracted using Radio-Immunoprecipitation Assay (RIPA) lysis buffer. Cell lysates were centrifuged at $16,000 \mathrm{rpm}$ for $15 \mathrm{~min}$ at $4^{\circ} \mathrm{C}$, and the protein concentration of the supernatant was measured using the BCA protein assay reagent (Pierce, California USA). Equal amounts of protein were separated by $12.5 \%$ or $15 \%$ SDS-PAGE and transferred onto nitrocellulose filter membranes. The membranes were blocked and incubated with the indicated antibodies. The protein bands were visualized using an IRDye 800CWconjugated secondary antibody (LI-COR Biosciences, Lincoln, $\mathrm{NE}$, USA), and the fluorescence signal was visualized using an Odyssey infrared imaging system (LI-COR Biosciences, Lincoln, NE, USA).

\section{Tissue microarray and immunohistochemistry}

A gastric cancer tissue microarray (ID:ST8014) was purchased from ChaoYing Biotechnology CO., LTD (Xian, China). The expression of EMC6 in the tissues was evaluated by immunohistochemical staining. Briefly, slides were incubated with an EMC6-specific antibody (1) overnight at $4^{\circ} \mathrm{C}$, followed by EnVisionTM/HRP (DAKO, Beijing, China) for $1 \mathrm{~h}$ at room temperature, then peroxidase reactivity was visualized using DAB substrate Kit (DAKO, Beijing, China). Finally, sections were counterstained with hematoxylin and observed.

\section{Tumorigenicity in nude mice}

A nude mouse xenograft model was established using 6-8 week-old female BALB/c nude mice (Experimental Animal Center, Peking University Health Sciences Center, Beijing, China). Mice were housed and maintained in a pathogen-free facility, and all experimental procedures were approved by the Institutional Authority for Laboratory Animal Care of Peking University. BGC823 cells stably transfected with pCDB-vector or pCDB-EMC6 were subcutaneously injected in the right axilla of BALB/C nude mice in a total volume of $100 \mu \mathrm{l}(4 \times$ $10^{6}$ cells). Mice were sacrificed $15-20$ days after cell inoculation. Tumors were excised and photographed.

\section{Statistical analysis}

Data are presented as the mean \pm SEM. Differences between groups were analyzed using Student's $t$ test for continuous variables. Statistical significance in this study was set at $\mathrm{P}<$ 0.05. All analyses were performed using GraphPad Prism 5.

\section{ACKNOWLEDGEMENTS}

This work was supported by Grants from the National Natural Sciences Foundation of China (31571052, 31370898 and
81420108002), the National Key Basic Research Program of China (973, 2011CB910103).

\section{CONFLICTS OF INTEREST}

The authors have no conflicting financial interests.

\section{REFERENCES}

1. Li Y, Zhao Y, Hu J et al (2013) A novel ER-localized transmembrane protein, EMC6, interacts with RAB5A and regulates cell autophagy. Autophagy 9, 150-163

2. Christianson JC, Olzmann JA, Shaler TA et al (2012) Defining human ERAD networks through an integrative mapping strategy. Nat Cell Biol 14, 93-105

3. Shen X, Kan S, Hu J et al (2016) EMC6/TMEM93 suppresses glioblastoma proliferation by modulating autophagy. Cell Death Dis 7, e2043

4. Richard $M$, Boulin T, Robert VJ, Richmond JE and Bessereau JL (2013) Biosynthesis of ionotropic acetylcholine receptors requires the evolutionarily conserved ER membrane complex. Proc Natl Acad Sci U S A 110, E1055- E1063

5. Szász AM, Lánczky A, Nagy Á et al (2016) Crossvalidation of survival associated biomarkers in gastric cancer using transcriptomic data of 1,065 patients. Oncotarget 7, 49322-49333

6. Klionsky DJ, Abdelmohsen K, Abe A et al (2016) Guidelines for the use and interpretation of assays for monitoring autophagy (3rd edition). Autophagy 12, 1-222

7. Jonikas MC, Collins SR, Denic V et al (2009) Comprehensive characterization of genes required for protein folding in the endoplasmic reticulum. Science 323, 1693-1697

8. Ruggiano A, Foresti O and Carvalho P (2014) Quality control: ER-associated degradation: protein quality control and beyond. J Cell Biol 204, 869-879

9. Wideman JG (2015) The ubiquitous and ancient ER membrane protein complex (EMC): tether or not? Version 2. F1000Res 4, 624

10. Lahiri S, Chao JT, Tavassoli S et al (2014) A conserved endoplasmic reticulum membrane protein complex (EMC) facilitates phospholipid transfer from the ER to mitochondria. PLoS Biol 12, e1001969

11. Satoh T, Ohba A, Liu Z, Inagaki T and Satoh AK (2015) $\mathrm{dPob} / \mathrm{EMC}$ is essential for biosynthesis of rhodopsin and other multi-pass membrane proteins in Drosophila photoreceptors. eLife 4, e06306

12. Bagchi $P$, Inoue $T$ and Tsai B (2016) EMC1-dependent stabilization drives membrane penetration of a partially destabilized non-enveloped virus. eLife 5, e21470

13. Harel T, Yesil G, Bayram Y et al (2016) Monoallelic and Biallelic Variants in EMC1 Identified in Individuals with Global Developmental Delay, Hypotonia, Scoliosis, and Cerebellar Atrophy. Am J Hum Genet 98, 562-570 